\title{
Impact of obesity and diabetes on the incidence of kidney and bladder cancers: a nationwide cohort study
}

\author{
Seung Hyun Ko ${ }^{1,2}$, Kyung Do Han³, Jae Seung Yun ${ }^{1,2}$, Sungjin Chung ${ }^{2,4}$ and Eun Sil Koh ${ }^{2,4}$ \\ 1'Division of Endocrinology and Metabolism, Department of Internal Medicine, St. Vincent's Hospital, College of \\ Medicine, The Catholic University of Korea, Gyeonggi-do, Republic of Korea, ${ }^{2}$ Department of Internal Medicine, \\ College of Medicine, The Catholic University of Korea, Seoul, Republic of Korea, ${ }^{3}$ Department of Biostatistics, College \\ of Medicine, The Catholic University of Korea, Seoul, Republic of Korea, and ${ }^{4}$ Division of Nephrology, Department of \\ Internal Medicine, Yeouido St. Mary's Hospital, College of Medicine, The Catholic University of Korea, Seoul, \\ Republic of Korea
}

Correspondence should be addressed to E S Koh

Email

fiji79@catholic.ac.kr

\begin{abstract}
Objective: Obesity and type 2 diabetes are becoming increasingly prevalent worldwide and are both associated with the increased incidence of kidney and bladder cancers. However, previous reports have provided conflicting results. We investigated the impact of body mass index (BMI) and diabetes on the incidence of both cancers in the general population.

Methods: Using nationally representative data from the Korean National Health Insurance System, 9,777,133 subjects without any malignancy who underwent health examinations in 2009 were followed to the end of 2017. Results: After a median follow-up period of 8.32 years, 12,544 incidents of kidney cancer and 15,137 incidents of bladder cancer were identified. The hazard ratio (HR) for kidney cancer was the lowest in people with a BMI $<18.5$ $\mathrm{kg} / \mathrm{m}^{2}$ (HR: $0.82,95 \%$ confidence interval (Cl): 0.72-0.94) and the highest in those with a BMI $\geq 30 \mathrm{~kg} / \mathrm{m}^{2}$ (HR: 1.71 , $95 \% \mathrm{Cl}: 1.57-1.87)$ compared to a reference BMI group $\left(18.5-23 \mathrm{~kg} / \mathrm{m}^{2}\right)$. In subjects with diabetes, obesity was associated with increased risk of kidney cancer, although the HRs were lower than observed in those without diabetes. Otherwise, there was a reduction in risk of bladder cancer with obesity in men and the HR for bladder cancer was not affected by BMI increase in women. There was a strongly positive association between diabetes and bladder cancer in the total study population.

Conclusions: Obesity was a strong risk factors for kidney cancer, whereas the association between obesity and bladder cancer differed by gender. The subjects with diabetes had a higher risk for both cancers than those without diabetes.
\end{abstract}

\section{Introduction}

Kidney and urinary bladder cancers are the most frequent malignant tumors of the urinary tract and comprise about $5 \%$ of all cancers worldwide (1). The kidney and bladder are essential organs that maintain the homeostatic balance of fluids and solutes and remove waste products from the blood. Further, the kidney also helps to regulate blood pressure and secretes several hormones. The link between metabolic abnormalities in obesity and type 2 diabetes (c) 2019 European Society of Endocrinology Printed in Great Britain
European Journal of Endocrinology

(2019) 181, 489-498 has been considered as a potential risk factor for these two cancers (2). Obesity and diabetes have been suggested to act synergistically, which aggravates inflammation, oxidative stress, hyperinsulinemia, and insulin resistance, and thus contributing to cancer development (3). Multiple previous epidemiologic studies and meta-analyses have been performed that have confirmed the associations between these cancers, obesity, and diabetes $(4,5,6,7,8,9)$.

Published by Bioscientifica Ltd. 
However, conflicting results have been presented in the literature. While obesity is an established risk factor for kidney cancer, diabetes has been not established as a risk factor. In terms of bladder cancer, type 2 diabetes is a distinct risk factor, but the evidence for obesity as a risk factor seems to be tenuous $(4,10,11)$. These results are unexpected and confusing (3), because cancer cells are influenced by the environmental nutrients that are regulated by the interactions between an individual's metabolic status and the distinct characteristics of cancer as influenced by the associations between obesity, diabetes, and cancer. This lack of consistency may be attributable to uncontrolled potential confounders, such as sex, hypertension, and several kinds of medications with the coexistence of obesity and diabetes.

To fill this relevant gap, we analyzed longitudinal nationwide cohorts with biennially updated covariate data derived from an adult general population to quantify the predictive role of body mass index (BMI) and the presence of diabetes on the risk for kidney and bladder cancers independent of key potential confounders.

\section{Methods}

\section{Data source and study population}

In this retrospective cohort study, we used the Korean National Health Insurance System (NHIS) database, which is government managed and the only insurer providing regular health check-up programs to the public in South Korea. Those enrolled in the health insurance service are recommended to undergo health check-ups at least biennially. In the present study, we included 10,490,501 subjects aged $\geq 20$ years who underwent a health examination between January 1, 2009 and December 31,2009 . Of these subjects, we excluded 152,309 having prior malignancy and 561,059 with missing data. This study was conducted according to the Declaration of Helsinki and approved by the Institutional Review Board of The Catholic University of Korea (No. SC18ZESI0154). Because anonymized and deidentified information was used in the analyses, informed consent was not required. The reporting of this study followed recommendations in the REporting studies Conducted using Observational Routinely collected health Data (RECORD) guidelines (12).

\section{Data collection}

Comorbidities were mainly defined using a combination of past history (ICD-10 code and self-reported) and use of medication history for the corresponding disease. The presence of hypertension was defined according to the presence of at least one claim per year under ICD10 codes I10 or I11 and at least one claim per year for the prescription of an antihypertensive agent or systolic/ diastolic blood pressure $\geq 140 / 90 \mathrm{mmHg}$. The presence of diabetes mellitus was defined according to the following criteria: (1) at least one claim per year under ICD-10 codes E10-14 and at least one claim per year for the prescription of antidiabetic medication or (2) fasting glucose level $\geq 126 \mathrm{mg} / \mathrm{dL}$. Blood samples for the measurement of serum glucose, total cholesterol, triglycerides, high-density lipoprotein cholesterol, and low-density lipoprotein cholesterol levels were drawn after fasting overnight. BMI was calculated as weight in kilograms divided by height in square meters. The Korean Society for the Study of Obesity recommends using BMI categories of underweight $(<18.5$ $\left.\mathrm{kg} / \mathrm{m}^{2}\right)$, normal weight $\left(18.5-22.9 \mathrm{~kg} / \mathrm{m}^{2}\right)$, overweight $\left(23-24.9 \mathrm{~kg} / \mathrm{m}^{2}\right)$, obese $\left(25-29.9 \mathrm{~kg} / \mathrm{m}^{2}\right)$, and morbidly obese $\left(>30 \mathrm{~kg} / \mathrm{m}^{2}\right)(13,14)$.

\section{Definition of kidney and bladder cancers and follow-up}

In this study, the primary outcome was newly diagnosed kidney or bladder cancers. Kidney and bladder cancers were coded as ICD-10 codes C64 and C67, respectively. Because the Korean Health Insurance Review and Assessment Service reimburses all medical care expenses for cancer, all cancer patients are registered in the database. Therefore, we could include each newly diagnosed cancer patient in the whole South Korean population. The study population was followed from baseline at the index year to the date of cancer diagnosis or December 31, 2017, whichever came first.

\section{Statistical analysis}

Baseline characteristics are presented as means \pm standard deviation, median value (interquartile range) or $n(\%)$. The incidence of primary outcomes was calculated by dividing the number of incident cases by the total follow-up duration (person-years). The disease-free probability of primary outcomes according to the combination with the presence of obesity and diabetes was calculated using Kaplan-Meier (K-M) curves, and a log-rank test was performed to analyze the differences among the groups. Hazard ratios (HRs) and 95\% confidence intervals (CIs) for kidney and bladder cancer were calculated using a Cox proportional hazards model for each category. Statistical 
analyses were performed using SAS software (v. 9.4; SAS Institute) and a $P$ value $<0.05$ was considered significant.

\section{Results}

\section{Characteristics of each group according to the presence of obesity and diabetes}

Table 1 shows the demographics of the study population according to the presence of obesity and diabetes. The proportion of patients with diabetes was $8.6 \%$ among a total of $9,777,133$ subjects, while the proportion of subjects with obesity (BMI $>25$ ) was $32.1 \%$. The percentages of subjects with obesity among nondiabetic and diabetic patients were 30.6 and $47.6 \%$, respectively. Compared with the DM/Obese (-/-) category, the other three categories had a higher proportion of men, alcohol drinkers, and patients of an older age, with higher blood pressure and dyslipidemia. There were more subjects with insulin use in the DM/Obese (+/-) category than in the DM/Obese $(+/+)$ category $(9.43$ vs $7.5 \%, P<0.001)$, whereas there were more patients with thiazolidinedione use, a potential risk factor for bladder cancer (15), in the DM/Obese $(+/+)$ category than in the DM/Obese $(+/-)$ category ( 7.9 vs $9.42 \%, P<0.001)$. The population with duration of diabetes over 5 years was larger in the DM/ Obese (+/-) category than in the DM/Obese (+/+) category.

\section{Risk of kidney and bladder cancers stratified by $\mathrm{BMI}$ across the presence of diabetes}

To analyze the impact of BMI on the incidence of kidney cancer, we divided the population into five groups according to their BMI and calculated the HR using subjects with a normal BMI range $\left(18.5-23 \mathrm{~kg} / \mathrm{m}^{2}\right)$ as a reference group (Table 2). In the total population, a markedly increasing HR for kidney cancer was observed in higher BMI categories: 1.23 (95\% CI 1.18-1.30), 1.43 (95\% CI 1.37-1.50), and 1.71 (95\% CI 1.57-1.87), respectively. In subjects with diabetes, obesity was associated with increased risk of kidney cancer, although the HRs were lower than observed in those without diabetes. In four categories divided by the presence of obesity and diabetes, the incidence rate of kidney cancer showed a remarkable increase in subjects with diabetes, regardless of combined obesity in the K-M curve (Fig. 1A). In subgroups without diabetes, the subjects in DM/Obese (-/+) had a higher incidence rate of kidney

Table 1 Demographics of the study population divided by the presence of obesity and diabetes. Data are expressed as the mean \pm S.D., the median value (interquartile range) or $n(\%)$.

\begin{tabular}{|c|c|}
\hline & DM/Obese (-/-) \\
\hline & $n=6,196,021$ \\
\hline Age, years & $45.35 \pm 14.1$ \\
\hline Male, $n(\%)$ & $3,118,623(50.33)$ \\
\hline $\mathrm{BMI}, \mathrm{kg} / \mathrm{m}^{2}$ & $21.95 \pm 1.98$ \\
\hline Waist circumference, cm & $76.1 \pm 7.25$ \\
\hline Body weight, kg & $59.05 \pm 8.66$ \\
\hline Height, $\mathrm{cm}$ & $163.73 \pm 8.98$ \\
\hline Fasting glucose, $\mathrm{mg} / \mathrm{dL}$ & $91.49 \pm 11.24$ \\
\hline Systolic BP, mmHg & $119.6 \pm 14.35$ \\
\hline Diastolic BP, mmHg & $74.65 \pm 9.65$ \\
\hline Total cholesterol, mg/dL & $191.21 \pm 35.2$ \\
\hline Triglyceride, mg/dL & 99.73 (99.69-99.77) \\
\hline HDL cholesterol, mg/dL & $57.41 \pm 18.34$ \\
\hline LDL cholesterol, mg/dL & $112.09 \pm 46.88$ \\
\hline Current smoker, $n(\%)$ & $1,568,509(25.31)$ \\
\hline Heavy drinker, $n(\%)$ & $362,961(5.86)$ \\
\hline Physical activity, $n(\%)$ & $3,120,185(50.36)$ \\
\hline Income lower $25 \%, n(\%)$ & $1,673,213(27)$ \\
\hline Hypertension, $n(\%)$ & $1,082,863(17.48)$ \\
\hline Dyslipidemia, $n(\%)$ & $797,438(12.87)$ \\
\hline Statin use, $n(\%)$ & $364,130(5.88)$ \\
\hline Insulin use, $n(\%)$ & . \\
\hline Thiazolinedione use, $n(\%)$ & . \\
\hline Duration of DM $>5$ years, $n(\%)$ & . \\
\hline
\end{tabular}

\begin{tabular}{c}
\hline DM/Obese $(-/+)$ \\
\hline$n=2,735,462$ \\
\hline $47.59 \pm 13.21$ \\
$1,722,452(62.97)$ \\
$27.28 \pm 2.06$ \\
$87.93 \pm 6.86$ \\
$74.31 \pm 10.33$ \\
$164.77 \pm 9.7$ \\
$94.8 \pm 11.77$ \\
$126.69 \pm 14.33$ \\
$79.16 \pm 9.81$ \\
$203.13 \pm 36.67$ \\
$140.27(140.18-140.36)$ \\
$52.02 \pm 18.78$ \\
$119.8 \pm 45.46$ \\
$788,427(28.82)$ \\
$239,552(8.76)$ \\
$1,472,087(53.81)$ \\
$682,301(24.94)$ \\
$936,671(34.24)$ \\
$637,145(23.29)$ \\
$309,557(11.32)$ \\
$\cdot$ \\
$\cdot$ \\
$\cdot$
\end{tabular}

\begin{tabular}{c}
\hline DM/Obese $(+/-)$ \\
\hline$n=442,898$ \\
\hline $58.22 \pm 12.1$ \\
$275,689(62.25)$ \\
$22.61 \pm 1.78$ \\
$80.79 \pm 6.52$ \\
$59.76 \pm 8.22$ \\
$162.32 \pm 8.96$ \\
$146.49 \pm 47.43$ \\
$127.42 \pm 15.84$ \\
$77.83 \pm 10.04$ \\
$194.47 \pm 41.23$ \\
$137.28(137.04-137.51)$ \\
$52.98 \pm 21.14$ \\
$110.09 \pm 45.08$ \\
$122,745(27.71)$ \\
$36,256(8.19)$ \\
$215,326(48.62)$ \\
$122,516(27.66)$ \\
$225,451(50.9)$ \\
$163,686(36.96)$ \\
$126,378(28.53)$ \\
$41,775(9.43)$ \\
$34,972(7.9)$ \\
$152,902(34.52)$ \\
\end{tabular}

\begin{tabular}{c}
\hline DM/Obese $(+/+)$ \\
\hline$n=402,752$ \\
\hline $56.26 \pm 11.9$ \\
$244,365(60.67)$ \\
$27.69 \pm 2.34$ \\
$90.68 \pm 7.04$ \\
$73.43 \pm 10.3$ \\
$162.62 \pm 9.43$ \\
$144.7 \pm 43.71$ \\
$131.06 \pm 15.31$ \\
$80.53 \pm 10.05$ \\
$200.31 \pm 41.91$ \\
$166.92(166.63-167.21)$ \\
$50.45 \pm 20.39$ \\
$111.87 \pm 46.4$ \\
$99,136(24.61)$ \\
$37,293(9.26)$ \\
$198,262(49.23)$ \\
$107,770(26.76)$ \\
$259,583(64.45)$ \\
$184,957(45.92)$ \\
$142,158(35.3)$ \\
$30,219(7.5)$ \\
$37,929(9.42)$ \\
$114,615(28.46)$ \\
\end{tabular}

BMI, body mass index; BP, blood pressure; HDL, high-density lipoprotein; LDL, low-density lipoprotein 
Table 2 Risk of kidney and bladder cancers stratified by BMI across the presence of diabetes.

\begin{tabular}{|c|c|c|c|c|c|c|}
\hline \multirow[b]{2}{*}{ BMI, $\mathrm{kg} / \mathrm{m}^{2}$} & \multirow[b]{2}{*}{$n$} & \multirow[b]{2}{*}{ Event } & \multirow[b]{2}{*}{$\mathbf{I R}^{*}$} & \multicolumn{3}{|c|}{ HR $(95 \% \mathrm{Cl})$} \\
\hline & & & & Model 1 & Model 2 & Model 3 \\
\hline \multicolumn{7}{|l|}{ Total } \\
\hline \multicolumn{7}{|l|}{ Kidney cancer } \\
\hline$<18.5$ & 371,850 & 217 & 0.072 & $0.78(0.68,0.90)$ & $0.78(0.68,0.90)$ & $0.82(0.72,0.94)$ \\
\hline $18.5-22.9$ & $3,847,566$ & 3451 & 0.109 & 1 (ref.) & 1 (ref.) & 1 (ref.) \\
\hline $23.0-24.9$ & $2,419,503$ & 3374 & 0.169 & $1.31(1.25,1.38)$ & $1.31(1.25,1.37)$ & $1.23(1.18,1.30)$ \\
\hline $25.0-29.9$ & $2,799,338$ & 4874 & 0.211 & $1.60(1.53,1.67)$ & $1.60(1.53,1.67)$ & $1.43(1.37,1.50)$ \\
\hline$\geq 30$ & 338,876 & 628 & 0.225 & $2.05(1.88,2.23)$ & $2.05(1.88,2.23)$ & $1.71(1.57,1.87)$ \\
\hline \multicolumn{7}{|c|}{ Bladder cancer } \\
\hline$<18.5$ & 371,850 & 421 & 0.14 & $0.85(0.76,0.94)$ & $0.77(0.70,0.85)$ & $0.76(0.69,0.85)$ \\
\hline $18.5-22.9$ & $3,847,566$ & 5153 & 0.163 & 1 (ref.) & 1 (ref.) & 1 (ref.) \\
\hline $23.0-24.9$ & $2,419,503$ & 4176 & 0.209 & $1.11(1.06,1.15)$ & $1.11(1.07,1.16)$ & $1.03(0.98,1.07)$ \\
\hline $25.0-29.9$ & $2,799,338$ & 4951 & 0.214 & $1.11(1.06,1.15)$ & $1.11(1.07,1.15)$ & $0.97(0.93,1.01)$ \\
\hline$\geq 30$ & 338,876 & 436 & 0.156 & $1.02(0.93,1.13)$ & $1.02(0.92,1.12)$ & $0.84(0.76,0.92)$ \\
\hline \multicolumn{7}{|l|}{ Non-diabetes } \\
\hline \multicolumn{7}{|c|}{ Kidney cancer } \\
\hline$<18.5$ & 359,523 & 198 & 0.068 & $0.80(0.69,0.92)$ & $0.79(0.69,0.92)$ & $0.82(0.71,0.95)$ \\
\hline $18.5-22.9$ & $3,634,326$ & 2998 & 0.1 & 1 (ref.) & 1 (ref.) & 1 (ref.) \\
\hline $23.0-24.9$ & $2,202,172$ & 2831 & 0.155 & $1.32(1.26,1.39)$ & $1.32(1.25,1.39)$ & $1.25(1.19,1.32)$ \\
\hline $25.0-29.9$ & $2,455,369$ & 3981 & 0.196 & $1.64(1.56,1.71)$ & $1.63(1.55,1.71)$ & $1.48(1.41,1.55)$ \\
\hline$\geq 30$ & 280,093 & 498 & 0.215 & $2.16(1.97,2.38)$ & $2.16(1.96,2.37)$ & $1.85(1.68,2.04)$ \\
\hline \multicolumn{7}{|c|}{ Bladder cancer } \\
\hline$<18.5$ & 359,523 & 389 & 0.133 & $0.89(0.81,1.00)$ & $0.82(0.73,0.91)$ & $0.78(0.70,0.87)$ \\
\hline $18.5-22.9$ & $3,634,326$ & 4381 & 0.146 & 1 (ref.) & 1 (ref.) & 1 (ref.) \\
\hline $23.0-24.9$ & $2,202,172$ & 3322 & 0.182 & $1.08(1.03,1.13)$ & $1.08(1.04,1.14)$ & $1.01(0.97,1.06)$ \\
\hline $25.0-29.9$ & $2,455,369$ & 3758 & 0.185 & $1.07(1.02,1.12)$ & $1.07(1.03,1.12)$ & $0.96(0.92,1)$ \\
\hline$\geq 30$ & 280,093 & 283 & 0.122 & $0.89(0.79,1.01)$ & $0.89(0.79,1.00)$ & $0.76(0.68,0.86)$ \\
\hline \multicolumn{7}{|l|}{ Diabetes } \\
\hline \multicolumn{7}{|c|}{ Kidney cancer } \\
\hline$<18.5$ & 12,327 & 19 & 0.216 & $0.77(0.49,1.23)$ & $0.78(0.49,1.24)$ & $0.83(0.52,1.31)$ \\
\hline $18.5-22.9$ & 213,240 & 453 & 0.269 & 1 (ref.) & 1 (ref.) & 1 (ref.) \\
\hline $23.0-24.9$ & 217,331 & 543 & 0.309 & $1.15(1.01,1.30)$ & $1.15(1.01,1.30)$ & $1.10(0.97,1.25)$ \\
\hline $25.0-29.9$ & 343,969 & 893 & 0.319 & $1.26(1.13,1.42)$ & $1.27(1.13,1.42)$ & $1.18(1.05,1.32)$ \\
\hline$\geq 30$ & 58,783 & 130 & 0.27 & $1.36(1.12,1.66)$ & $1.38(1.13,1.68)$ & $1.22(1.00,1.49)$ \\
\hline \multicolumn{7}{|c|}{ Bladder cancer } \\
\hline$<18.5$ & 12,327 & 32 & 0.363 & $0.74(0.52,1.05)$ & $0.73(0.51,1.03)$ & $0.75(0.53,1.07)$ \\
\hline $18.5-22.9$ & 213,240 & 772 & 0.458 & 1 (ref.) & 1 (ref.) & 1 (ref.) \\
\hline $23.0-24.9$ & 217,331 & 854 & 0.487 & $1.07(0.97,1.18)$ & $1.08(0.98,1.19)$ & $1.06(0.96,1.17)$ \\
\hline $25.0-29.9$ & 343,969 & 1193 & 0.426 & $1.02(0.93,1.12)$ & $1.03(0.94,1.13)$ & $0.99(0.91,1.09)$ \\
\hline$\geq 30$ & 58,783 & 153 & 0.318 & $1.15(0.96,1.36)$ & $1.16(0.98,1.38)$ & $1.09(0.91,1.30)$ \\
\hline
\end{tabular}

$\mathrm{BMI}$, body mass index; $\mathrm{Cl}$, confidence interval; $\mathrm{HR}$, hazard ratio.

Model 1, adjusted for age and sex; model 2, adjusted for model 1 plus smoking, drinking, exercise and income; model 3, adjusted for model 2 plus diabetes, hypertension and dyslipidemia in an analysis of the total population; model 3, adjusted for model 2 plus hypertension and dyslipidemia in a subgroup analysis according to the presence of diabetes.

*Incidence rate per 1000 person-years.

cancer than those in DM/Obese $(-/-)(P$ values by logrank $<0.001)$.

In the general population the HR for bladder cancer was not affected as BMI increased (Table 2). In four categories divided by the presence of obesity and diabetes, the incidence rate of bladder cancer showed a remarkable increase in the subgroup with diabetes. Interestingly, the subjects in DM/Obese (+/-) showed a higher incidence rate for bladder cancer than those in DM/Obese (+/+) (Fig. 1B). The subjects without diabetes had a lower incidence rate of bladder cancer than those with diabetes regardless of the presence of obesity.

\section{Incidence rates and the risk of kidney and bladder cancers according to BMI category and the presence of diabetes}

We divided the subjects into ten categories in order to using combinations of five BMI ranges and the presence of diabetes and investigated the risk for two cancers using 

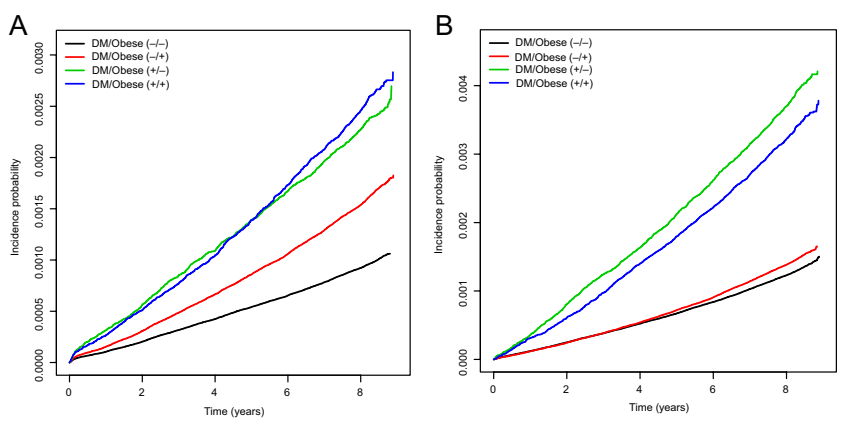

Figure 1

Kaplan-Meier estimates of cumulative incidence. (A) Kidney and $(B)$ bladder cancers were among four categories divided by the presence of obesity and diabetes. The analysis was performed in an unadjusted model and both $P$ values by log-rank were $<0.001$.

a nondiabetic category with BMI $18.5-23 \mathrm{~kg} / \mathrm{m}^{2}$ as a reference as shown in Fig. 2. When stratified by serial BMI categories as shown in Fig. 2A, the HR for kidney cancer showed sequential increments from the subgroup without diabetes: 0.83 (95\% CI: 0.72-0.96),1 (reference), 1.25 (95\% CI: 1.19-1.32), 1.48 (95\% CI: 1.41-1.55), 1.86 (95\% CI: $1.69-2.04)$; to the subgroup with diabetes: $1.06(95 \%$ CI: 0.67-1.66), 1.30 (95\% CI: 1.18-1.44), 1.53 (95\% CI: 1.42-1.66), and 1.59 (95\% CI: 1.33-1.89), respectively. In the subgroup analysis divided by gender (Fig. 2B and C), the impact of obesity (BMI $>25 \mathrm{~kg} / \mathrm{m}^{2}$ ) on kidney cancer risk was not significant in women with diabetes.

For bladder cancer, the incidence rate and HR did not show sequential increments as BMI increased (Fig. 2D). However, the subjects with diabetes had a higher HR for bladder cancer compared with those without diabetes regardless of their BMI. Also, the association between obesity and bladder cancer risk differed by gender. In men without diabetes, there was a reduction in risk of bladder cancer with obesity; while in those with diabetes, there was a similar trend but it was not statistically significant (Fig. 2E). The results were distinguished from those in women (Fig. 2F). In addition, diabetic women with morbid obesity (BMI $\left.>30 \mathrm{~kg} / \mathrm{m}^{2}\right)$ had a HR of 2.31 (95\% CI 1.71-3.11) for bladder cancer compared with nondiabetic women with normal BMI.

\section{Subgroup analyses and the risk of kidney cancer according to BMI}

To evaluate the effect modifiers affecting the relationships between obesity, diabetes and kidney cancer, we conducted a stratified analysis using several factors such
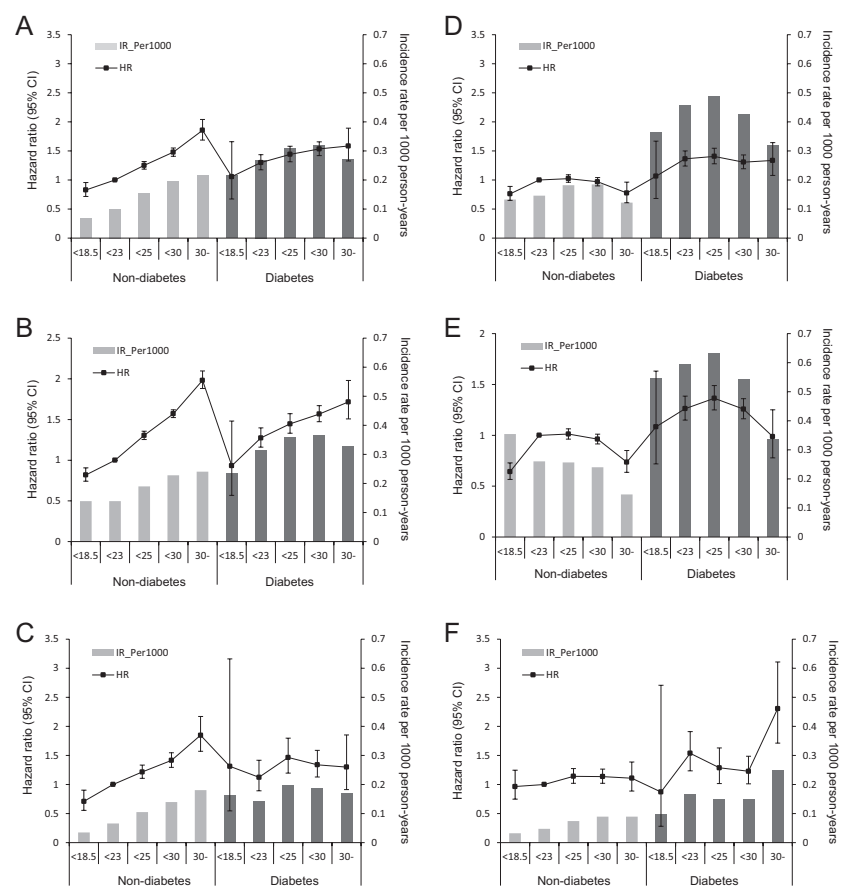

\section{Figure 2}

Incidence rates and hazard ratios ( $95 \%$ confidence intervals) of kidney cancer and bladder cancer stratified by BMI and the presence of diabetes across gender. Ten categories were divided by combinations of five BMI ranges and the presence of diabetes compared with a reference nondiabetic category with BMI $18.5-23 \mathrm{~kg} / \mathrm{m}^{2}$. Risk of kidney cancer in the total study population (A), in men (B) and in women (C). Risk of bladder cancer in the total study population (D), in men (E) and in women (F). Adjusted for age, sex, smoking, alcohol drinking, regular exercise, income status, presence of hypertension, and dyslipidemia (A and D). Adjusted for age, smoking, alcohol drinking, regular exercise, income status, presence of hypertension, and dyslipidemia (B,C,E and F).

as age, sex, smoking status, presence of hypertension and dyslipidemia (Table 3). Only in subgroup without diabetes, the risk for kidney cancer according to BMI was significantly different with stratification by age $(P$ for interaction, 0.003). Further, the risk of kidney cancer according to BMI was different between subjects with insulin treatment and those without, although it might not be statistically significant ( $P$ for interaction, 0.19). Thus, in subjects with diabetes not using insulin therapy, the HR for kidney cancer was affected by an increase in BMI: 0.81 (95\% CI: 0.48-1.36), 1 (reference), 1.18 (95\% CI: 1.03-1.35), 1.24 (95\% CI: 1.09-1.40), 1.31 (95\% CI: 1.07-1.63); however, it was not affected by BMI in subjects with diabetes using insulin therapy. 


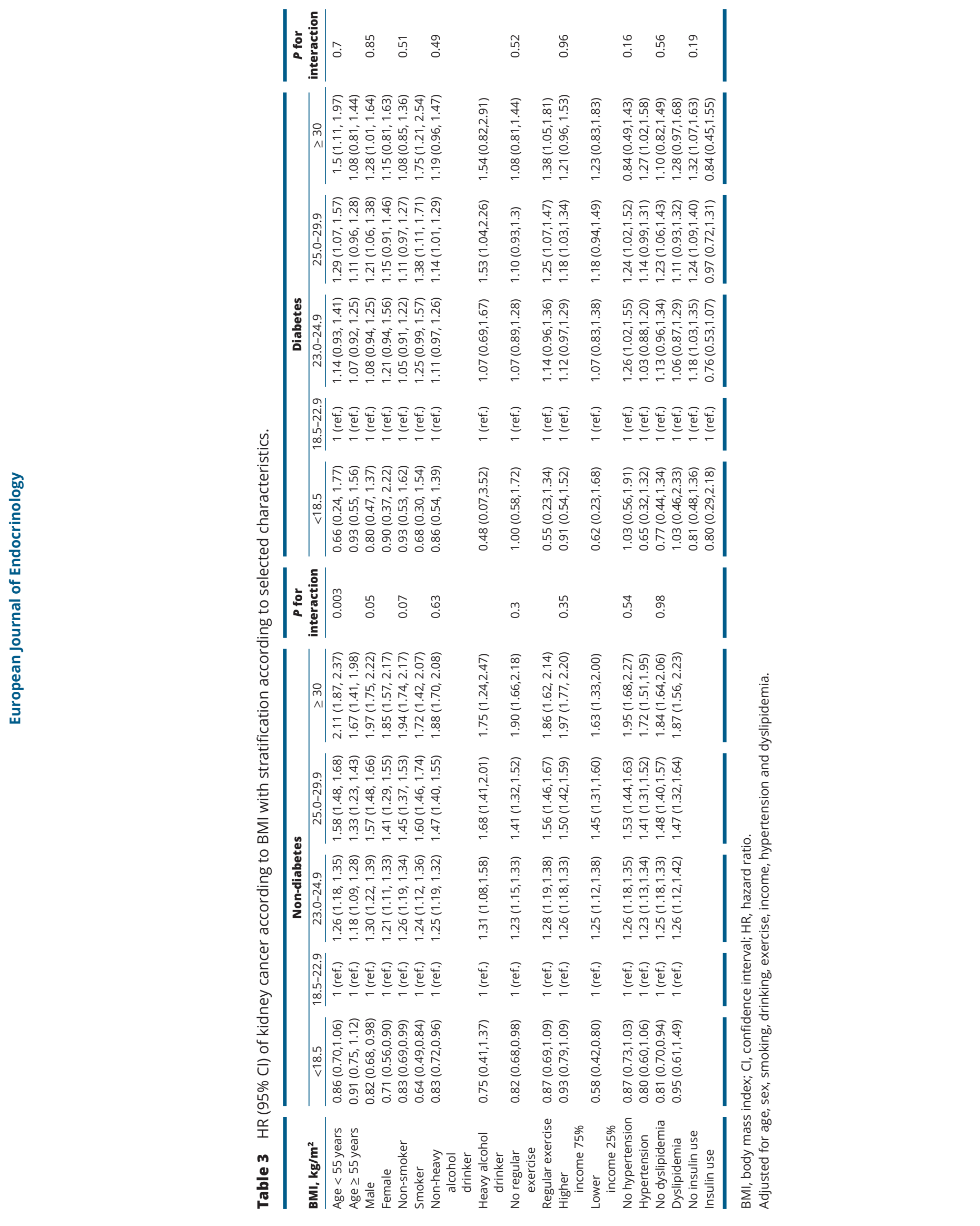




\section{Subgroup analyses and the risk of bladder cancer according to BMI}

In subgroups without diabetes divided according to gender, smoking status, alcohol habits, exercise, income, presence of hypertension and dyslipidemia, the association between BMI and bladder cancer risk was different (Table 4). Interestingly, in subjects without hypertension, the HR of subjects with a BMI $<18.5 \mathrm{~kg} / \mathrm{m}^{2}$ was $1.34(95 \%$ CI: $1.18-1.51$ ) and that of subjects with a BMI $>30 \mathrm{~kg} / \mathrm{m}^{2}$ was 0.65 (95\% CI: 0.53-0.81) compared with subjects with a normal BMI $\left(18.5-23 \mathrm{~kg} / \mathrm{m}^{2}\right)$. Thus, underweight subjects without hypertension had a higher risk of bladder cancer than those with a normal BMI. Further, men without diabetes had a negative association between obesity and bladder cancer risk. In the subgroups with diabetes, the association between BMI and bladder cancer risk differed only when divided according to gender ( $P$ for interaction $<0.0001$ ). Diabetic women with morbid obesity (BMI $>30$ $\mathrm{kg} / \mathrm{m}^{2}$ ) had a HR of 1.63 (95\% CI: $\left.1.20-2.22\right)$ for bladder cancer compared with those with normal BMI.

\section{Discussion}

The present study demonstrated the impact of obesity and diabetes on the incidence of kidney and bladder cancers after adjusting for several confounding factors in the nationwide Korean population. Subjects with diabetes had a higher risk for both cancers than those without diabetes. Overweight and obesity were strong risk factors for kidney cancer, whereas they were not for bladder cancer. Thus, in subjects with diabetes, obesity was associated with increased risk of kidney cancer, although the HRs were lower than those observed in subjects without diabetes. The risk of bladder cancer was higher in subjects with diabetes than in those without diabetes regardless of BMI. Further, the associations between obesity, diabetes and kidney and bladder cancer risk were affected by gender. The impact of obesity on kidney cancer was relatively weak in subjects with diabetes, especially in women. In men, the risk of bladder cancer tended to reduce as BMI increased regardless of the presence of diabetes. Our findings provide distinct information regarding the associations between obesity and kidney and bladder cancer risks across the presence of diabetes and gender in a nationwide population.

The biological mechanism for obesity-related carcinogenesis is not yet well characterized; however, many possibilities have been suggested: for example, obesity leads to diverse hormonal alterations and involves the insulin/insulin-like growth factor (IGF) pathway, sex steroids, and adipokines $(5,6,16)$. In particular, hyperinsulinemia can be considered as an independent carcinogenic factor that increases the amount of proinflammatory cytokines, reactive oxygen species, and lipid peroxidation. Insulin acts as a growth factor for tumor cell mitosis and stimulates IGF-1 activation (17). Insulin and IGF-1 share a major downstream regulation pathway and both are engaged in carcinogenesis (18). These enhanced insulin/IGF-1 pathways by obesity and diabetes can be a plausible mechanism to explain the current results of kidney cancer. However, in a case of bladder cancer, our present data showed an obscure association with BMI and bladder cancer, which was inconsistent with several previous studies that provided evidence for such a link between IGF and bladder cancer $(19,20)$. These different impacts on the incidence of both cancers across the presence of diabetes can partially be explained by the distinct activation of restricted organ- or cell-specific insulin/IGF-1 signaling pathways $(3,4,21)$.

Another proposed mechanism underlying the relationships between obesity, diabetes, and cancer can be explained by an increased aromatase activity in adipose tissue, which thereby converts androgens to estrogens. Estrogens can activate IGF-1 receptor expression and may affect cell proliferation and growth. Estrogen receptor expression has been linked to cancer initiation and progression in bladder cancer $(22,23)$ and is attributable to abnormal secretion of higher estrogen levels among individuals with obesity (7). Previous studies showed a different incidence of both cancers based on gender (24, 25). Graff et al. reported that diabetes was independently associated with a greater risk of kidney cancer in women but not in men (10). Chen et al. showed that men with diabetes had significantly higher hazard of bladder cancer than men without diabetes, but not in women (26). Our results also demonstrated this discrepancy by gender. In the subgroup with diabetes in the present study, the impact of obesity on kidney cancer risk was significant in the total population but not in women with diabetes. For bladder cancer, a rather negative association between BMI and incidence rate for bladder cancer was observed in men without diabetes, which was distinguished from that of women without diabetes. Furthermore, diabetic women with morbid obesity had a 2.3-fold risk for bladder cancer compared to those with normal BMI. The present heterogenous results by gender implies that sex hormone implicates carcinogenesis of kidney and bladder with hormonal alteration related to postmenopausal effect or use of oral contraceptives in women. The exact mechanism by which sex hormone exerts its carcinogenic effect remains to be determined. 


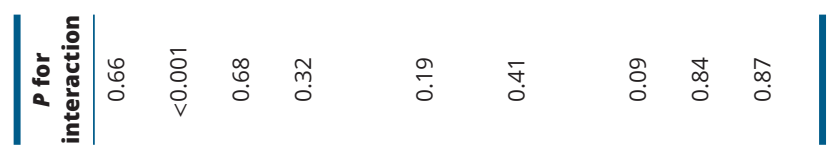
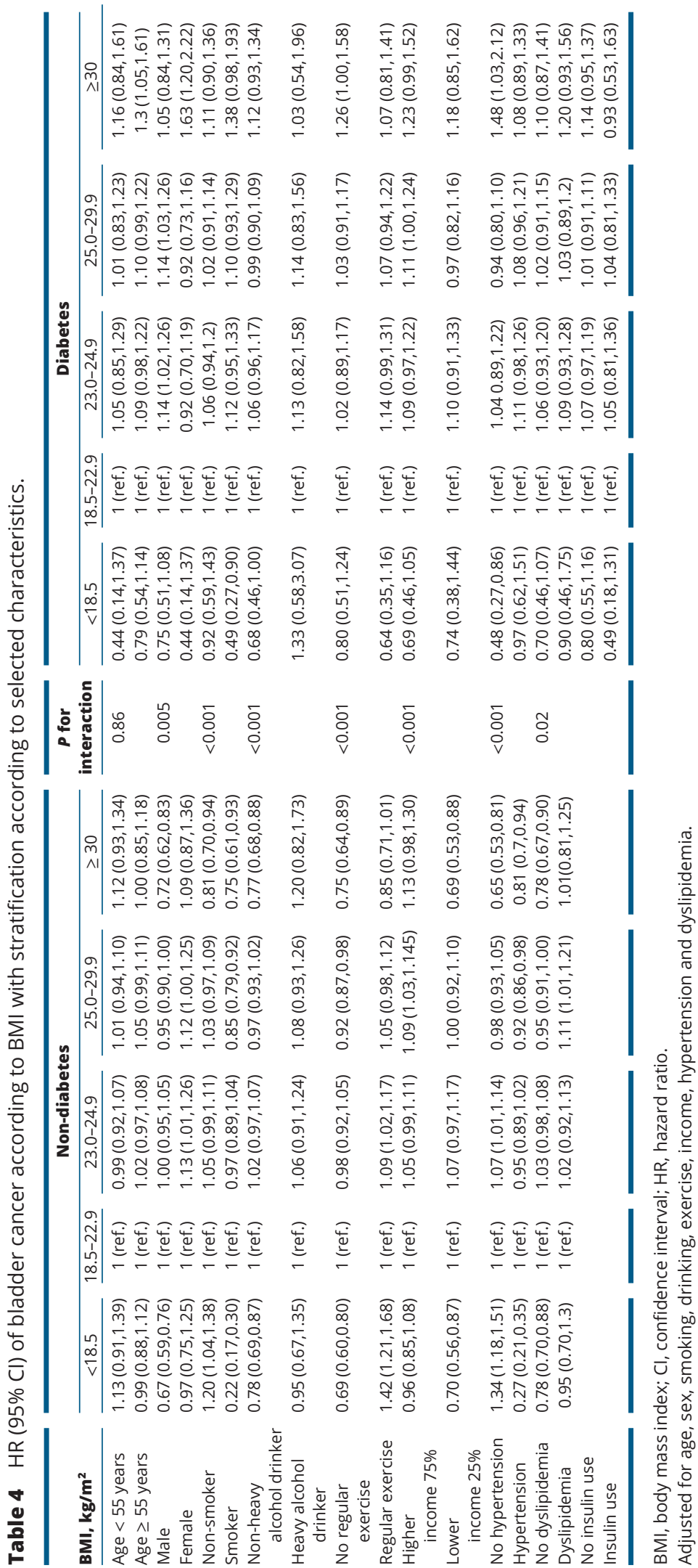
One of the interesting details of the current data was in the subgroup with diabetes study of their risk for kidney cancer. Whether they received insulin treatment or not, the HR showed a different trend for the development of kidney cancer. The subjects not using insulin therapy had an independently higher risk for kidney cancer with higher BMI, which was consistent with the results for the total study participants. However, the subjects using insulin therapy were not affected by BMI categories. We can assume that exogenous insulin leads to hyperinsulinemia, which can promote the activation of IGF-1-signaling pathway; however, in real-world clinical practice, there has been no evidence that insulin treatment increases the risk of kidney cancer. In terms of bladder cancer, a previous in vitro study suggested that high-dose human insulin treatment may promote bladder cancer cell proliferation $(27,28,29)$. However, the present study did not show a difference in the impact on bladder cancer by BMI stratification across insulin use in a subgroup with diabetes. Therefore, an association with endogenous insulin hypersecretion may not be translated into a pathway for the use of exogenous insulin in individuals with diabetes.

Another interesting detail from the results was the different incidences of bladder cancer between the DM/ Obese (+/-) and DM/Obese (+/+) categories. There was a reduction in risk of bladder cancer with obesity especially in men. Previous studies reported that overweight and obesity were confusing risk factors for bladder cancer, which may be due to two strong risk factors, that is, smoking and diabetes. Smoking is generally associated with lower BMI and unhealthy behaviors of men. BMI was not considered as a risk factor for bladder cancer in a recent bladder cancer guideline and our data were consistent with this result $(11,23)$. Meanwhile, the positive association between diabetes and bladder cancer could be explained by the mitogenic properties of insulin and diabetic patients' susceptibility to urinary tract infections, which warrants further investigation $(8,30,31)$.

Our study had several limitations. First, we followed an observational design; therefore, inferences of causality should not be made regarding obesity and diabetes with the development of two cancers. Second, we had limited information to define a pathologic classification of tumor heterogeneity (32). Third, the subgroups with diabetes varied in the ascertainment of the status and inclusion of patients with type 1 diabetes. Fourth, our cohort comprised primarily Korean participants and our results need to be validated in a more-diverse population of patients. Finally, we could not directly assess the severity of type 2 diabetes and did not have reliable information on the diverse combinations of medications used. Despite these limitations, this is a nationally representative, populationbased study evaluating the association of obesity and diabetes as risk factors for kidney and bladder cancers, and the results verify the possibility of a potentially protective effect of combining individual weight reduction and tight glucose control by either medications or lifestyle modifications to decrease the risk for these two cancers. A future intervention study could help elucidate these two risk factors for kidney and bladder cancer outcomes.

In conclusion, we observed that diabetes was an independent risk factor for developing kidney and bladder cancers in a nationwide Korean general populationbased cohort study. Obesity was a risk factor for kidney cancer, but not for bladder cancer. These findings support the strategies of encouraging an active surveillance for the high risk subjects and modulating the metabolic derangement in the general population to prevent the development of these two cancers.

\section{Declaration of interest}

The authors declare that there is no conflict of interest that could be perceived as prejudicing the impartiality of the research reported.

\section{Funding}

This research was supported by a grant of the Korea Health Technology R\&D Project through the Korea Health Industry Development Institute (KHIDI), funded by the Ministry of Health \& Welfare, Republic of Korea (grant number: HI18C0275).

\section{Author contribution statement}

SHK, KDH and ESK conceived and designed the study. KDH performed a statistical analysis and ESK wrote the manuscript. SHK, KDH, JSY, SC and ESK contributed to discussion.

\section{Acknowledgements}

This study was performed using the database from the National Health Insurance System (NHIS-2019-1-127), and the results do not necessarily represent the opinion of the National Health Insurance Corporation.

\section{References}

1 Ferlay J, Soerjomataram I, Dikshit R, Eser S, Mathers C, Rebelo M, Parkin DM, Forman D \& Bray F. Cancer incidence and mortality worldwide: sources, methods and major patterns in GLOBOCAN 2012. International Journal of Cancer 2015136 E359-E386. (https:// doi.org/10.1002/ijc.29210)

2 Chow WH, Dong LM \& Devesa SS. Epidemiology and risk factors for kidney cancer. Nature Reviews Urology 20107 245-257. (https://doi. org/10.1038/nrurol.2010.46)

3 Gallagher EJ \& LeRoith D. Obesity and diabetes: the increased risk of cancer and cancer-related mortality. Physiological Reviews 201595 727-748. (https://doi.org/10.1152/physrev.00030.2014) 
4 Chow WH, Gridley G, Fraumeni JF, Jr. \& Jarvholm B. Obesity, hypertension, and the risk of kidney cancer in men. New England Journal of Medicine 2000343 1305-1311. (https://doi.org/10.1056/ NEJM200011023431804)

5 Luo J, Margolis KL, Adami HO, Lopez AM, Lessin L, Ye W \& Women's Health Initiative Investigators. Body size, weight cycling, and risk of renal cell carcinoma among postmenopausal women: the Women's Health Initiative (United States). American Journal of Epidemiology 2007 166 752-759. (https://doi.org/10.1093/aje/kwm137)

6 Joh HK, Willett WC \& Cho E. Type 2 diabetes and the risk of renal cell cancer in women. Diabetes Care 201134 1552-1556. (https://doi. org/10.2337/dc11-0132)

7 Liu X, Sun Q, Hou H, Zhu K, Wang Q, Liu H, Zhang Q, Ji L \& Li D. The association between BMI and kidney cancer risk: an updated dose-response meta-analysis in accordance with PRISMA guideline. Medicine (Baltimore) 201897 e12860. (https://doi.org/10.1097/ MD.0000000000012860)

8 MacKenzie T, Zens MS, Ferrara A, Schned A \& Karagas MR. Diabetes and risk of bladder cancer: evidence from a case-control study in New England. Cancer 2011117 1552-1556. (https://doi.org/10.1002/ cncr.25641)

9 Noguchi JL, Liss MA \& Parsons JK. Obesity, physical activity and bladder cancer. Current Urology Reports 201516 74. (https://doi org/10.1007/s11934-015-0546-2)

10 Graff RE, Sanchez A, Tobias DK, Rodriguez D, Barrisford GW, Blute ML, Li Y, Sun Q, Preston MA, Wilson KM et al. Type 2 diabetes in relation to the risk of renal cell carcinoma Among men and women in two large prospective cohort studies. Diabetes Care 2018 41 1432-1437. (https://doi.org/10.2337/dc17-2518)

11 Prizment AE, Anderson KE, Yuan JM \& Folsom AR. Diabetes and risk of bladder cancer among postmenopausal women in the Iowa Women's Health Study. Cancer Causes \& Control 201324 603-608. (https://doi.org/10.1007/s10552-012-0143-3)

12 Benchimol EI, Smeeth L, Guttmann A, Harron K, Moher D, Petersen I, Sørensen HT, von Elm E, Langan SM \& RECORD Working Committee. The REporting of studies Conducted using Observational Routinely-collected health Data (RECORD) Statement. PLOS Medicine 201512 e1001885. (https://doi.org/10.1371/journal.pmed.1001885)

13 WHO Expert Consultation. Appropriate body-mass index for Asian populations and its implications for policy and intervention strategies. Lancet 2004363 157-163. (https://doi.org/10.1016/S01406736(03)15268-3)

14 Obesity: preventing and managing the global epidemic. Report of a WHO consultation. World Health Organization Technical Report Series 2000894 i-xii, 1, 1-253.

15 Tseng CH. Pioglitazone and bladder cancer: a population-based study of Taiwanese. Diabetes Care 201235 278-280. (https://doi. org/10.2337/dc11-1449)

16 Labochka D, Moszczuk B, Kukwa W, Szczylik C \& Czarnecka AM. Mechanisms through which diabetes mellitus influences renal cell carcinoma development and treatment: a review of the literature. International Journal of Molecular Medicine 201638 1887-1894. (https://doi.org/10.3892/ijmm.2016.2776)

17 Oh Y. The insulin-like growth factor system in chronic kidney disease: pathophysiology and therapeutic opportunities. Kidney Research \& Clinical Practice 201231 26-37. (https://doi.org/10.1016/j. krcp.2011.12.005)

18 Tracz AF, Szczylik C, Porta C \& Czarnecka AM. Insulin-like growth factor-1 signaling in renal cell carcinoma. BMC Cancer 201616453. (https://doi.org/10.1186/s12885-016-2437-4)

19 Xu T, Zhu Z, Wang X, Xia L, Zhang X, Zhong S, Sun F, Zhu Y \& Shen $\mathrm{Z}$. Impact of body mass on recurrence and progression in
Chinese patients with Ta, T1 urothelial bladder cancer. International Urology and Nephrology 201547 1135-1141. (https://doi.org/10.1007/ s11255-015-1013-1)

20 Cantiello F, Cicione A, Salonia A, Autorino R, De Nunzio C, Briganti A, Gandaglia G, Dell'Oglio P, Capogrosso P \& Damiano R. Association between metabolic syndrome, obesity, diabetes mellitus and oncological outcomes of bladder cancer: a systematic review. International Journal of Urology 201522 22-32. (https://doi. org/10.1111/iju.12644)

21 Zhu S, Xu F, Zhang J, Ruan W \& Lai M. Insulin-like growth factor binding protein-related protein 1 and cancer. Clinica Chimica Acta; International Journal of Clinical Chemistry 2014431 23-32. (https:// doi.org/10.1016/j.cca.2014.01.037)

22 Choi W, Porten S, Kim S, Willis D, Plimack ER, Hoffman-Censits J, Roth B, Cheng T, Tran M, Lee IL et al. Identification of distinct basal and luminal subtypes of muscle-invasive bladder cancer with different sensitivities to frontline chemotherapy. Cancer Cell 201425 152-165. (https://doi.org/10.1016/j.ccr.2014.01.009)

23 Gild P, Ehdaie B \& Kluth LA. Effect of obesity on bladder cancer and renal cell carcinoma incidence and survival. Current Opinion in Urology 201727 409-414. (https://doi.org/10.1097/ MOU.0000000000000425)

24 Beebe-Dimmer JL, Colt JS, Ruterbusch JJ, Keele GR, Purdue MP, Wacholder S, Graubard BI, Davis F, Chow WH \& Schwartz KL. Body mass index and renal cell cancer: the influence of race and sex. Epidemiology 201223 821-828. (https://doi.org/10.1097/ EDE.0b013e31826b7fe9)

25 Antoni S, Ferlay J, Soerjomataram I, Znaor A, Jemal A \& Bray F. Bladder cancer incidence and mortality: a global overview and recent trends. European Urology 201771 96-108. (https://doi.org/10.1016/j. eururo.2016.06.010)

26 Chen HF, Chen SW, Chang YH \& Li CY. Risk of malignant neoplasms of kidney and bladder in a cohort study of the diabetic population in Taiwan With age, sex, and geographic area stratifications. Medicine (Baltimore) 201594 e1494. (https://doi.org/10.1097/ MD.0000000000001494)

27 Liu S, Li Y, Lin T, Fan X, Liang Y \& Heemann U. High dose human insulin and insulin glargine promote T24 bladder cancer cell proliferation via PI3K-independent activation of Akt. Diabetes Research \& Clinical Practice 201191 177-182. (https://doi. org/10.1016/j.diabres.2010.11.009)

28 Metalli D, Lovat F, Tripodi F, Genua M, Xu SQ, Spinelli M, Alberghina L, Vanoni M, Baffa R, Gomella LG et al. The insulin-like growth factor receptor I promotes motility and invasion of bladder cancer cells through Akt- and mitogen-activated protein kinasedependent activation of paxillin. American Journal of Pathology 2010 176 2997-3006. (https://doi.org/10.2353/ajpath.2010.090904)

29 Tseng CH. Human insulin does not increase bladder cancer risk. PLOS ONE 20149 e86517. (https://doi.org/10.1371/journal. pone.0086517)

30 Fang H, Yao B, Yan Y, Xu H, Liu Y, Tang H, Zhou J, Cao L, Wang W, Zhang J et al. Diabetes mellitus increases the risk of bladder cancer: an updated meta-analysis of observational studies. Diabetes Technology \& Therapeutics 201315 914-922. (https://doi.org/10.1089/ dia.2013.0131)

31 Tseng CH. Diabetes and risk of bladder cancer: a study using the National Health Insurance database in Taiwan. Diabetologia 201154 2009-2015. (https://doi.org/10.1007/s00125-011-2171-z)

32 Hsieh JJ, Purdue MP, Signoretti S, Swanton C, Albiges L, Schmidinger M, Heng DY, Larkin J \& Ficarra V. Renal cell carcinoma. Nature Reviews. Disease Primers 20173 17009. (https://doi. org/10.1038/nrdp.2017.9) 